\title{
Genetically Engineered Gold-Binding Polypeptides: Structure Prediction and Molecular Dynamics
}

\author{
Rosemary Braun* Mehmet Sarikaya†, and Klaus Schulten*
}

November 16, 2001

*Beckman Institute \& Department of Physics, University of Illinois, Urbana 61801, USA

${ }^{\dagger}$ Materials Science \& Engineering, University of Washington, Seattle, WA 98195, USA 


\begin{abstract}
The biological control of inorganic crystal formation, morphology and assembly is of interest to biologists and biotechnologists studying hard tissue growth and regeneration, as well as to materials scientists using biomimetic approaches for control of inorganic material fabrication and assembly. Biomimetics requires an accurate understanding of natural mechanisms at the molecular level. Such understanding can be derived from the use of metal surfaces to study surface recognition by proteins together with combinatorial genetics techniques for selection of suitable peptides. Polymerization of these peptides produces engineered polypeptides large enough to encode their own folding information with low structural complexity while enhancing binding affinity to surfaces. The low complexity of such polypeptides can aid in analyses leading to modeling and eventual manipulation of the structure of the folded polypeptide. Here we present structure predictions for gold-binding protein sequences, originally selected by combinatorial techniques. Molecular dynamics simulations lasting 5 ns were carried out using solvated polypeptides at the gold surface to assess the dynamics of the binding process and the effects of surface topography on the specificity of protein binding.
\end{abstract}




\section{Introduction}

Many biological hard tissues (bones, dentin, enamel, spicules, particles, and spines) contain proteins in addition to inorganic minerals [1]. Proteins are essential in the formation of biological materials through their control of nucleation, growth, morphogenesis, phase organization and distribution [2, 3, 4]. Genetic engineering techniques (cell-surface display [5] and phage display [6]) have lead to the isolation of simple proteins with specific binding affinity to selected practical inorganics. Using these proteins as building blocks opens avenues to the engineering of materials with novel properties.

A recent example of this genetic approach is furnished by gold-binding proteins (GBPs). These proteins were selected for the ability to bind to gold in the presence of high salt concentrations, conditions under which other proteins do not exhibit gold binding [5, 7]. During the selection processes, it was found that the gold-binding polypeptides bind more strongly to gold after the surface is treated with HF to remove surface impurities, indicating that the GBPs recognize the native gold surface rather than a partially removed contaminant. Additionally, GBPs preferentially bind gold over chromium, demonstrating substrate specificity [5].

The GBP sequences contain multiple repeats of a 14-30 amino acid sequence [5, 7]. The repeating polypeptides retain their binding properties as part of other proteins (e.g., alkaline phosphatase) if they contain a sufficient number of repeats, and affinity for gold increases with the number of repeats. Interestingly, none of the GBP sequences contain cysteine, which is known to form a covalent bond to gold [8]. All the GBP sequences to date have contained methionine. The experimental literature is inconclusive as to whether methionine can form a covalent bond to gold $[9,10]$; however, because the protein was released from the surface in the presence of a detergent, and because other polypeptides containing methionine did not bind to gold, it is unlikely that the methionine sulfur contributes to the binding. Whereas many proteins and self-assembled monolayers bind to gold via disulfide bonds $[11,12,13]$ the nature of GBP binding is thought to differ from this well-known thiol linkage and offer a new avenue for protein-gold surface interaction. The GBP sequences are all found to be rich in serine and threonine, and physisorption of these polar sidechains onto gold may account for the observed binding.

In addition to their binding characteristics, GBPs alter gold crystal morphology under ambient conditions [7]. In the presence of GBP, gold formed large, flat hexagonal crystals displaying the $\{111\}$ face. Such crystals were not seen to form in the presence of control proteins that do not bind to gold; using the well-known Faraday approach, flat crystals only form under boiling or highly acidic conditions [14]. A possible cause of flat-gold formation may be the preferential binding onto the $\mathrm{Au}\{111\}$ surface, obscuring it from further gold accretion. It is not readily apparent how the GBP adheres to gold, nor why the $\{111\}$ surface would be preferred to, e.g., the more sparsely populated $\{211\}$ face.

Knowledge of the structure of GBP could provide insight into the mechanism by which GBP binds to gold. Molecular dynamics simulations of the predicted structure, which can

give a detailed description of the dynamics over the course of several nanoseconds, could shed light on the interaction of GBP with various crystal surfaces. These simulations also could elucidate the role of water in the GBP-gold interaction. 


\section{Methods}

\section{Structure Prediction}

Although amino acid sequences were known for three GBPs (Table 1), there was no experimental structural information. Secondary structure prediction of the GBPs was carried out without a priori knowledge of the binding characteristics of the proteins using a combination of neural network [15] and sequence alignment methods in addition to ab initio structure modelling. The first two sequences (GBP1 and GBP2) consisted of a 14 amino acid sequence repeated six times (84 residues); the third (GBP3) consisted of two different 14 amino acid sequences each repeated approximately 3.5 times (94 residues).

A combination of statistical and sequence alignment methods were used for structure prediction for the three GBPs studied here. JPred [15] combines the results from several neural networks (DSC, PHD, PREDATOR, and others) and multiple sequence alignments (PSIBlast, ClustalW). Each of the neural networks were trained on a set of at least 126 proteins, and the results are considered to be $\sim 70 \%$ accurate. However, since there are very few homologs to the GBP sequences, the less accurate ab initio structure predictor ROSETTA [16] was used as an additional check.

Once predictions were obtained, QUANTA [17] was used to map several repeats of the GBP1 sequence onto an antiparallel $\beta$-sheet. PROCHECK [18] was used to check for bad angles, contacts, etc. The resulting structure was minimized using X-PLOR [19].

\section{Molecular Dynamics}

To simulate the protein in contact with gold, two Au surfaces, displaying the $\{111\}$ face and the $\{211\}$ face, were created manually based on the known gold FCC crystal structure and lattice spacing of $4.07 \AA$. The Au surfaces were $5 \AA$ (3 layers) deep and approximately $40 \AA$ by $80 \AA$ wide. Coordinates for the GBP were taken from the minimized predicted structure. The GBP was manually positioned on each of the gold surfaces. A pre-equilibrated water box was overlayed on the protein-metal system, and overlapping waters were subsequently removed, as well as waters between the GBP and Au surface. The complete system was comprised of 594 protein atoms, 1457 (672) Au atoms for the $\{111\}(\{211\})$ surface, and approximately 13,000 water atoms, bringing the total to approximately 15,000 atoms.

Molecular dynamics simulations were carried out using the program NAMD2 [20], with the CHARMM26 force field [21]. Additional terms were necessary in order to model the metal surface. It is known that a single water molecule physisorbs onto a metal surface with an energy of between 7 and $15 \mathrm{kcal} / \mathrm{mol}$ which, while stronger than hydrogen bond energy $(5 \mathrm{kcal} / \mathrm{mol})$, is almost as large as the interaction energy between water molecules in bulk water under ambient conditions $(19.8 \mathrm{kcal} / \mathrm{mol})$ [22]. At the interface, a compromise between the forces leading to physisorption and solvation can be expected. Typically, the interfacial structure of the water decays to bulklike properties within a few solvent diameters; on the metal side, the electron density and surface structure decay to bulklike arrangements at yet shorter distances [23]. The interaction between the metal (which is rigid but with a diffuse electronic structure) and the adsorbates (which exhibit incoherent particle motion 
and localized electronic structure) may be represented by a model potential in cases where the metal's properties are not the focus of interest. The overall potential between a molecule and a metal surface, $U_{\text {surf }}$, may be represented [24] by a Lennard-Jones 10-4 potential,

$$
U_{\text {surf }}=2 \pi \sum_{l=0}^{\infty} n_{l} \epsilon \sigma^{2}\left[\frac{2}{5}\left(\frac{\sigma}{z+d_{l}}\right)^{10}-\left(\frac{\sigma}{z+d_{l}}\right)^{4}\right]
$$

where $d_{l}$ is the separation between the first and $l$ th lattice planes, $n_{l}$ is the number density of atoms in plane $l$, and $z$ is the perpendicular separation of the adsorbate particle from the first $(l=0)$ lattice plane. The parameters $\sigma$ and $\epsilon$ are the Lennard-Jones $6-12$ potential parameters describing the interaction between the adsorbate and surface atoms. Because there is no significant contribution from below the second lattice plane, only the first two terms of the sum need be considered. The corrugation of the more sparsely populated (eg, $\{211\}$ ) planes may be mimicked by making the $d_{l}$ 's sinusoidal functions [23]. For Au, $\sigma$ and $\epsilon$ are $1.8474 \AA$ and $0.0390 \mathrm{kcal} / \mathrm{mol}$, respectively [25].

In addition to the metal-adsorbate interactions, it is of interest to consider the electrostatic potential contribution due to the interaction of molecular point charges with the induced image charge "beneath" the metal plane. For a smooth surface, this contribution is given by

$$
U_{\text {surf }}^{\mathrm{img}}\left(z_{1}, \ldots, z_{n}\right)=\frac{\epsilon_{m}-\epsilon_{s}}{\epsilon_{m}\left(\epsilon_{m}+\epsilon_{s}\right)}\left[\sum_{i} \frac{q_{i}^{2}}{4 z_{i}}+\frac{1}{2} \sum_{\substack{i, j \\ i \neq j}} \frac{q_{i} q_{j}}{\left|\vec{r}_{i j}+2 \vec{n} z_{j}\right|}\right]
$$

where the first term describes the interaction of a charge $q_{i}$ at a distance $z_{i}$ above the image plane (generally defined to be the plane in which the nuclei of the atoms in the topmost lattice plane lie) with its own image charge, and the second term describes the interaction between a charge and the images of all other charges. $\vec{r}_{i j}$ denotes the separation between real charges, $\vec{n}$ is the unit vector perpendicular to the image plane, and the $\epsilon$ 's are the dielectric constant for the surface $\left(\epsilon_{s}=\infty\right)$ and the medium $\left(\epsilon_{m}=80\right.$, water $)$, respectively. However, for small distances $z$ above the gold plane, the Lennard-Jones contribution dominates the image-charge contribution, whereas for large $z$, neither term contributes significantly. Furthermore, it has been observed that, for a neutral many-particle system, the sum of charge - image charge interactions is close to zero [26, 27]; such cancellation has been noted even with monolayer films of water. Thus, it is reasonable to exclude the charge - image charge terms from the Hamiltonian.

Molecular dynamics simulations using force fields of this type (a smoothed potential representing the metal surface and no charge-image contribution), have been shown to reproduce accurately the experimentally measurable surface concentrations of water [28] and hydrocarbons [29]. Explicitly including the polarizability has been shown not to affect the interfacial structure [28], since the induced dipole moment of a water molecule in the polarizable models tends to point in the same direction as the permanent moment. Additionally, molecular mechanics using the above model force field has been used to calculate the minimum energy monolayer of hydrocarbon chains on $\mathrm{MoSe}_{2}$; the resulting structure agrees well with the observed STM structure [29]. 
Molecular dynamics simulations were carried out, at time steps of $1 \mathrm{fs}$, using the program NAMD2 [19] in conjunction with the Au potential for both the $\{111\}$ and $\{211\}$ planes with the gold atoms held fixed to speed up computation. Each system was simulated with periodic boundary conditions, with full electrostatics computed using the Particle Mesh Ewald (PME) [30] method with a grid spacing on the order of $1 \AA$ or less. Each system was energy minimized using the Powell algorithm, then heated for 2 ps under Langevin dynamics at a temperature of $300 \mathrm{~K}$ and with a damping coefficient of $10 \mathrm{ps}^{-1}$. Each system was then equilibrated for $1 \mathrm{~ns}$ at constant pressure and temperature. Pressure was maintained at 1 atm using the Langevin piston method [31] while temperature coupling was enforced by velocity reassignment every 2 ps. The systems were then simulated for 4 ns further in the NpT ensemble using Langevin dynamics/Langevin piston at a temperature of $300 \mathrm{~K}$ and a damping coefficient of $10 \mathrm{ps}^{-1}$. Analysis of trajectories and energetics was performed using $\mathrm{X}-\mathrm{PLOR}[19]$ and VMD [32].

\section{Results}

Secondary structure prediction was carried out on three known GBP sequences, necessitated by lack of experimental structural data. For GBP1, there was agreement between several prediction methods indicating an anti-parallel $\beta$-sheet. For GBP2, the results for various methods differed, with some indicating helical regions where others predicted $\beta$-strands or coils. For GBP3, the neural network predictions did not arrive at any specific structure other than a random coil, and the similarity searches matched sequences of a globular character. Due to the ambiguity of GBP2 and the indeterminate structure for GBP3, we have confined our detailed studies to GBP1. Three repeats of the GBP1 14 amino-acid sequence were mapped onto the anti-parallel $\beta$-sheet structure suggested by the prediction tools, minimized, and checked for bad angles, contacts, etc.

The predicted GBP1 structure was placed on both $\{111\}$ and $\{211\}$ gold surfaces large enough to accommodate it and fully solvated; water molecules between the GBP and Au surface were removed. The system in its pre-equilibrium configuration is shown in Fig. 1. The stability of the predicted structure in contact with the gold surfaces can be assessed by analyzing its deviation from the initial structure over the course of the equilibration. Fig. 1 depicts the root mean square deviation (RMSD) of the $\mathrm{C}_{\alpha}$ atoms of the protein during equilibration. After $500 \mathrm{ps}$, the protein stabilizes at approximately $3 \AA$ RMSD from its pre-equilibrium structure. Because the initial structure was a predicted, rather than a crystallographic one, such deviation is expected. Four molecular dynamics simulations lasting 4 ns were carried out on each plane. No major conformational changes were observed. Each strand of the GBP adsorbs onto the $\{111\}$ Au plane with an energy of $\sim 9 \mathrm{kcal} / \mathrm{mol}$, roughly twice the adsorption energy of water onto the $\{111\}$ plane [28]. It is observed that the main contributions to the adsorption energy come from the polar amino acids rather than the hydrophobic ones (Fig. 2). Visual examination of the GBP position on the gold surfaces (Fig. 3) reveals that while the protein is composed equally of polar and hydrophobic residues, the atoms in close contact with the gold surface are primarily those from the polar sidechains. Additionally, the polar residues exhibited a much smaller deviation from their 
initial position than the nonpolar residues (Fig. 1), indicating stability with respect to the fixed gold lattice.

The adsorption energy of GBP onto the $\{211\}$ plane is much lower than that of GBP onto the $\{111\}$ plane (Fig. 2). The difference in lattice geometry between the $\{111\}$ and $\{211\}$ plane, i.e. the twofold higher density of atoms of the $\{111\}$ surface, is expected to result in a twofold reduction of the potential. However, the observed reduction in the adsorption energy on the $\{211\}$ plane cannot be fully accounted for by the lower density of gold atoms. Presence of water in the $\{211\}$ surface corrugations is believed to hinder interaction of the GBP with the Au surface, thereby contributing to face-specific binding. Although water molecules within $3 \AA$ of both GBP and the Au surface were removed at the beginning of the simulation, over the course of 4 ns water molecules diffused between the GBP and Au surface. While the main contribution to the water molecule count depicted in Fig. 4 is from water molecules at the edges of GBP, the difference between the $\mathrm{Au}\{111\}$ and $\mathrm{Au}\{211\}$ surface is primarily due to diffusion of water underneath GBP in the corrugations of the $\{211\}$ plane. A sample snapshot revealing the differences in water placement is shown in Fig. 5. The specific interaction of a single polypeptide with a crystallographic surface could be studied experimentally using AFM equipped with a functionalized tip and with modeling studies using steered molecular dynamics [33] simulations in which GBP is pulled across the gold surfaces under full solvation.

\section{Conclusions}

The predicted structure and molecular dynamics simulations of the engineered polypeptide selected for its gold binding characteristics explain the nature of the GBP-gold interaction as well as differences of interactions between $\mathrm{Au}\{111\}$ and $\{211\}$ crystal surfaces. The results of the model predict a periodic structure of hydroxyls on the surface of the antiparallel $\beta$ sheet that is conducive to binding to the Au lattice. This model is similar to electrostatic binding of organic matrix proteins to $\mathrm{CaCO}_{3}$ surfaces [4] and binding of small antifreeze proteins with tandem repeats onto ice crystal [34, 35]; in both cases, repetitive charged groups commensurate with the lattice spacing have been interpreted to be the cause of crystal binding. Results of the simulations of GBP1 in contact with gold surfaces show that the protein is stable. The close contacts to the gold surface come from the sidechains of the polar amino acids, consistent with the experimental observation that the proteins selected for their ability to bind to gold are richer in serine and threonine than those which fail to bind tightly to gold.

The nature of mineral/protein interaction is of great importance in many fields of biology and materials sciences, including biomineralization, inhibition or promotion of iceformation in organisms, and in assembly of functional inorganics using polypeptides. Engineered polypeptides have great potential as model systems in fundamental studies of protein interaction with inorganic crystals. These simple proteins may serve as a conceptual model for protein control of mineral formation in hard-tissue growth and tissue engineering, and as molecular erector sets in materials nanoassembly [36, 37]. Although spectroscopic work remains to be performed, extensive modeling as demonstrated here provides a first glimpse of 
the engineered protein conformation and binding to inorganic crystals, a significant step to-

wards molecular biomimetics via the exploitation of recognition capabilities and interactions found in biological systems [37].

\section{References}

[1] H.A. Lowenstam. Minerals formed by organisms. Science, 211:1126-1131, 1981.

[2] S. Mann. Molecular recognition in biomineralization. Nature, 332:119-121, 1988.

[3] M. Sarikaya. Biomimetics: Materials fabrication through biology. PNAS, 96:14183$14185,1999$.

[4] G. Fallini, S. Albeck, S. Weiner, and L. Addadi. Control of aragonite or calcite polymorphism by mollusk shell macromolecules. Science, 271:67-69, 1996.

[5] S. Brown. Metal recognition by repeating polypeptides. Nature Biotechnology, 15:269272, 1997.

[6] S. R. Whaley, D. S. English, E. L. Hu, P. F. Barbara, and A. M. Belcher. Selection of peptides with semiconducting binding specificity for directed nanocrystal assembly. Nature, 405:665-668, 2000.

[7] S. Brown, M. Sarikaya, and E. A. Johnson. A genetic analysis of crystal growth. J. Mol. Bio., 299:725-735, 2000.

[8] P. Fenter, A. Eberhardt, and P. Eisenberger. Self-assembly of n-alkyl thiols as disulfides on au(111). Science, 266:1216-1218, 1994.

[9] R. Brizzolara, J. Boyd, and A. Tate. Evidence for covalent attachment of purple membrane to a gold surface via genetic modification of bacteriorhodopsin. J. Vac. Sci. Technol. A, 15(3):773, 1997.

[10] J. A. Cuff, M. E. Clamp, A. S. Siddiqui, M. Finlay, and G. Barton. Jpred: A consensus secondary structure prediction server. Bioinformatics, 14:892-893, 1998.

[11] M. Tarek, K. Tu, M. Klein, and D. Tobias. Molecular dynamics simulations of supported phospholipid / alkanethiol bilayers on a gold(lll) surface. Biophysical Journal, 77:964, 1999.

[12] Z. Zhang and T. Beck. Molecular structure of monolayers from thiol-terminated polyimide model compounds on gold. Langmuir, 12:1227, 1995.

[13] J. Qian, R. Hentschke, and W. Knoll. Superstructures of cyclodextrin derivatives on au(111): a combined random planting - molecular dynamics approach. Langmuir, 13:7092, 1997. 
[14] J. Turkowitch, P.C Stevenson, and J. Hillier. A study of nucleation and growth progress in the synthesis of colloidal gold. Trans. Faraday Soc. Disc, 11:55-70, 1951.

[15] A. Strong and B. Moore. Self-assembling chiral monolayers of helical peptides bound to gold via side-chain thioethers. Chem. Commun., page 473, 1998.

[16] K. T. Simons, R. Bonneau, I. Ruczinski, and D. Baker. Ab initio protein structure prediction of casp iii targets using rosetta. Proteins, 37 Suppl 3:171-6, 1999.

[17] Molecular Simulations Inc., Burlington, Massachusetts. QUANTA 97, 1997.

[18] R. A. Laskowski, M. W. MacArthur, D. S. Moss, and J. M. Thornton. PROCHECK: a program to check the stereochemical quality of protein structures. J. Appl. Cryst., 26:283-291, 1993.

[19] Axel T. Brünger. X-PLOR, Version 3.1: A System for X-ray Crystallography and NMR. Yale University Press, New Haven and London, 1992.

[20] Laxmikant Kalé, Robert Skeel, Milind Bhandarkar, Robert Brunner, Attila Gursoy, Neal Krawetz, James Phillips, Aritomo Shinozaki, Krishnan Varadarajan, and Klaus Schulten. NAMD2: Greater scalability for parallel molecular dynamics. J. Comp. Phys., 151:283-312, 1999.

[21] A. D. MacKerell Jr., D. Bashford, M. Bellott, R. L. Dunbrack Jr., J. Evanseck, M. J. Field, S. Fischer, J. Gao, H. Guo, S. Ha, D. Joseph, L. Kuchnir, K. Kuczera, F. T. K. Lau, C. Mattos, S. Michnick, T. Ngo, D. T. Nguyen, B. Prodhom, I. W. E. Reiher, B. Roux, M. Schlenkrich, J. Smith, R. Stote, J. Straub, M. Watanabe, J. WiorkiewiczKuczera, D. Yin, and M. Karplus. All-hydrogen empirical potential for molecular modeling and dynamics studies of proteins using the CHARMM22 force field. J. Phys. Chem. B, 102:3586-3616, 1998.

[22] W. L. Jorgensen, J. Chandrasekhar, J. D. Madura, R. W. Impey, and M. L. Klein. Comparison of simple potential functions for simulating liquid water. J. Chem. Phys., 79:926-935, 1983.

[23] J. Shelley and D. Berard. Computer simulation of water physisorption at metal-water interfaces. Reviews in Computational Chemistry, 12:137, 1998.

[24] R. Hentschke. Macromol. Theory Simul., 6:287, 1997.

[25] A. Rappe, C. Casewitt, K. Colwell, W. Goddard, and W. Skiff. Uff, a rule-based full periodic table force field for molecular mechanics and molecular dynamics simulations. J. Am. Chem. Soc., 114:10024, 1992.

[26] J. Shelley, G. Patey, D. Berard, and G. Torrie. Modeling and structure of mercury-water interfaces. J. Chem. Phys., 107:2122, 1997. 
[27] J. Qian, R. Hentschke, and W. Knoll. Superstructures of cyclodextrin derivatives on au(111): a combined random planting - molecular dynamics approach. Langmuir, 13:7092, 1997.

[28] J. Shelley and D. Berard. Computer simulation of water physisorption at metal-water interfaces. Reviews in Computational Chemistry, 12:137, 1998.

[29] R. Hentschke. Molecular modeling of adsorption and ordering at solid interfaces. Macromol. Theory Simul., 6:287, 1997.

[30] T.A. Darden, D.M. York, and L.G. Pedersen. Particle mesh Ewald. An N.log(N) method for Ewald sums in large systems. J. Chem. Phys., 98:10089-10092, 1993.

[31] S.E. Feller, Y.H. Zhang, R.W. Pastor, and B.R. Brooks. Constant pressure molecular dynamics simulation - the Langevin piston method. J. Chem. Phys., 103(11):4613-4621, 1995.

[32] William F. Humphrey, Andrew Dalke, and Klaus Schulten. VMD - Visual Molecular Dynamics. J. Mol. Graphics, 14:33-38, 1996.

[33] Sergei Izrailev, Sergey Stepaniants, Manel Balsera, Yoshi Oono, and Klaus Schulten. Molecular dynamics study of unbinding of the avidin-biotin complex. Biophys. J., 72:1568-1581, 1997.

[34] C. A. Knight, C. C. Cheng, and A. L. DeVries. Adsorption of alpha-helical antifreeze peptides on specific ice crystal surface planes. Biophys. J., 59:409-411, 1991.

[35] Y-C. Liou, A. Tocilj, P. Davies, and J. Zhongchao. Mimicry of ice structure by surface hydroxyls and water of alpha-helix antifreeze proteins. Nature, 406:322-324, 2000.

[36] C.Mirkin and T. Taton. Semiconductors meet biology. Nature, 405:626-627, 2000.

[37] P. Ball. Life's lesson in design. Nature, 409:413-416, 2001.

\section{Acknowledgements}

The authors wish to thank Ferenc Molnar and James Phillips (University of Illinois), Stanley Brown (University of Copenhagen), and Daniel Heidel (University of Washington) for discussions. The work of RB and KS was supported by the National Institutes of Health (NIH PHS 5 P41 RR05969), the Roy J. Carver Charitable Trust, and NRAC grant MCA935028. The work of MS was supported by ARO-DURINT.

Correspondence and requests should be addressed to KS (kschulte@ks.uiuc.edu). 


\section{Table 1}

GBP1 MHGKTQATSGTIQSMHGKTQATSGTIQSMHGKTQATSGTIQSMHGKTQATSGTIQSMHGK

....EEEEEEEEEEE . . . EEEEEEEEE . . . EEEEEEEEE . . . EEEEEEEEE . .

GBP2 ALVPTAHRLDGNMHALVPTAHRLDGNMHALVPTAHRLDGNMHALVPTAHRLDGNMHALVP

$\ldots \ldots$. НHH $\ldots \ldots$. . НHHHH . . . . . . НHHHH . . . . . НHHHH . . . . . НH .

GBP3 LQATPGMKMRLSGAKEATPGMKMRLSGAKEATPGMSTTVAGLLQATPGMKMRLSGAKEAT



Table 1: GBP sequences and corresponding consensus structure predictions. Data is truncated after 60 amino acids. In the prediction output, E signifies a $\beta$-strand, $\mathrm{H}$ signifies a helix, and . signifies random coil. Confidence in the prediction is highest for GBP1. 


\section{Figures}

Figure 1: RMSD of $C_{\alpha}$ atoms during the equilibration relative to the predicted starting structure (black). The protein is stable after 500ps. Polar residues (green) exhibit small RMSD compared to the fluctuations observed in the hydrophobic residues (red). The inset depicts the initial $\beta$-sheet configuration of GBP.

Figure 2: Adsorption energies. In (a), GBP on Au $\{111\}$ is shown as a solid line while GBP on $\mathrm{Au}\{211\}$ is shown as dotted. In (b), the solid line shows the average contribution to the adsorption energy on the $\{111\}$ plane from Ser and Thr, whereas the dashed line shows the average contribution from all other residues.

Figure 3: GBP on (a) $\{111\}$ and (b) $\{211\}$ Au surface, viewed from above (top) and edge-on (bottom). Atoms near the surface are shown as spheres. Coloring corresponds to residue type: polar residues are highlighted in blue, charged are in green, and hydrophobic are in red.

Figure 4: Number of water molecules within $3 \AA$ of both GBP and the Au plane. The data is smooted by taking a running average over a 20 ps window. Data for the $\{111\}$ plane is shown as a solid line; data for the $\{211\}$ plane is shown as a dotted line.

Figure 5: Water molecules between GBP and Au after 2 ns. The dark shadow represents the GBP. Water molecules beneath the GBP are highlighted in blue. Only one is seen on the $\{111\}$ surface (a) in contrast to the four molecules seen in the corrugations of the $\{211\}$ surface (b). 


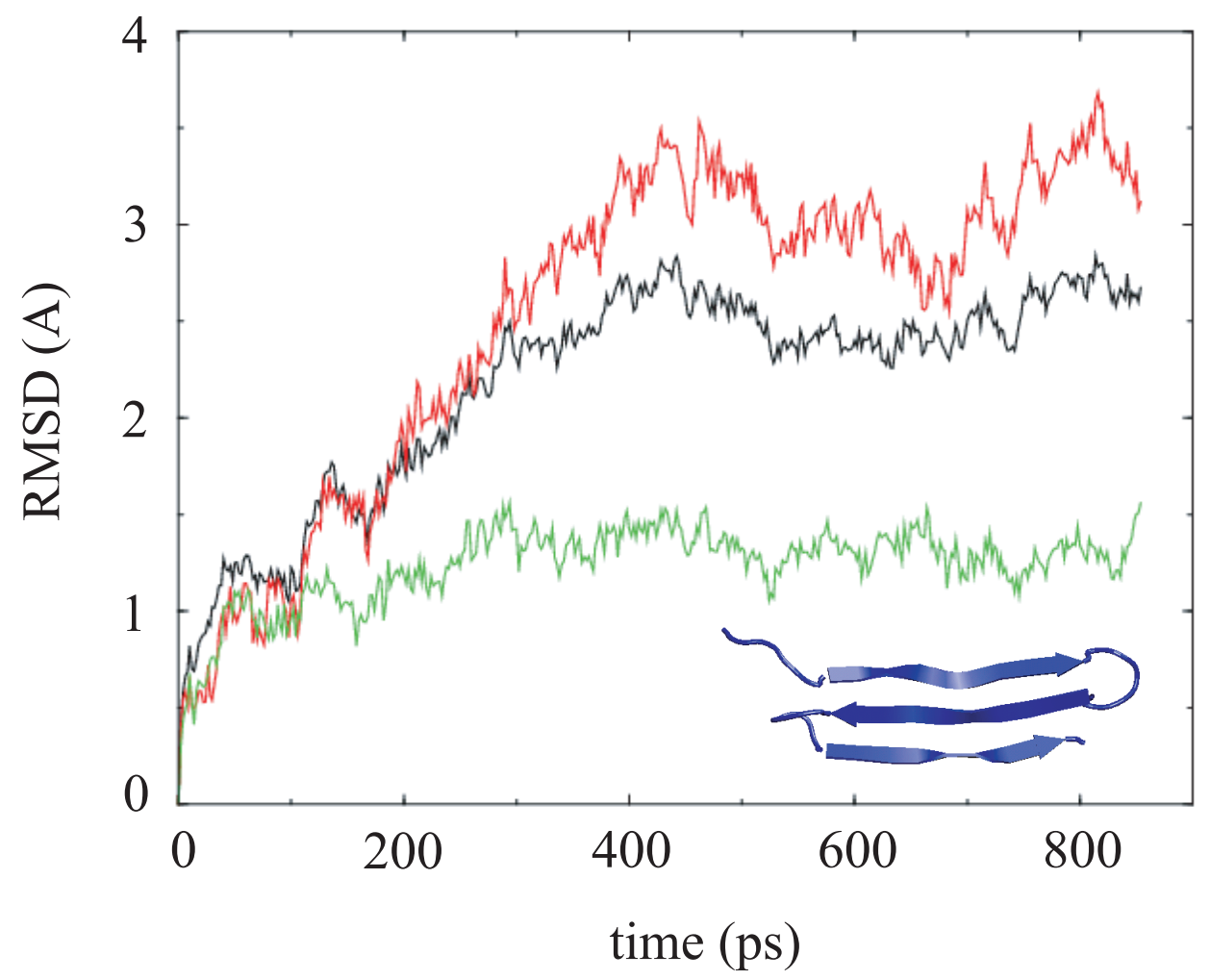

Braun, Sarikaya \& Schulten. Figure 1. 


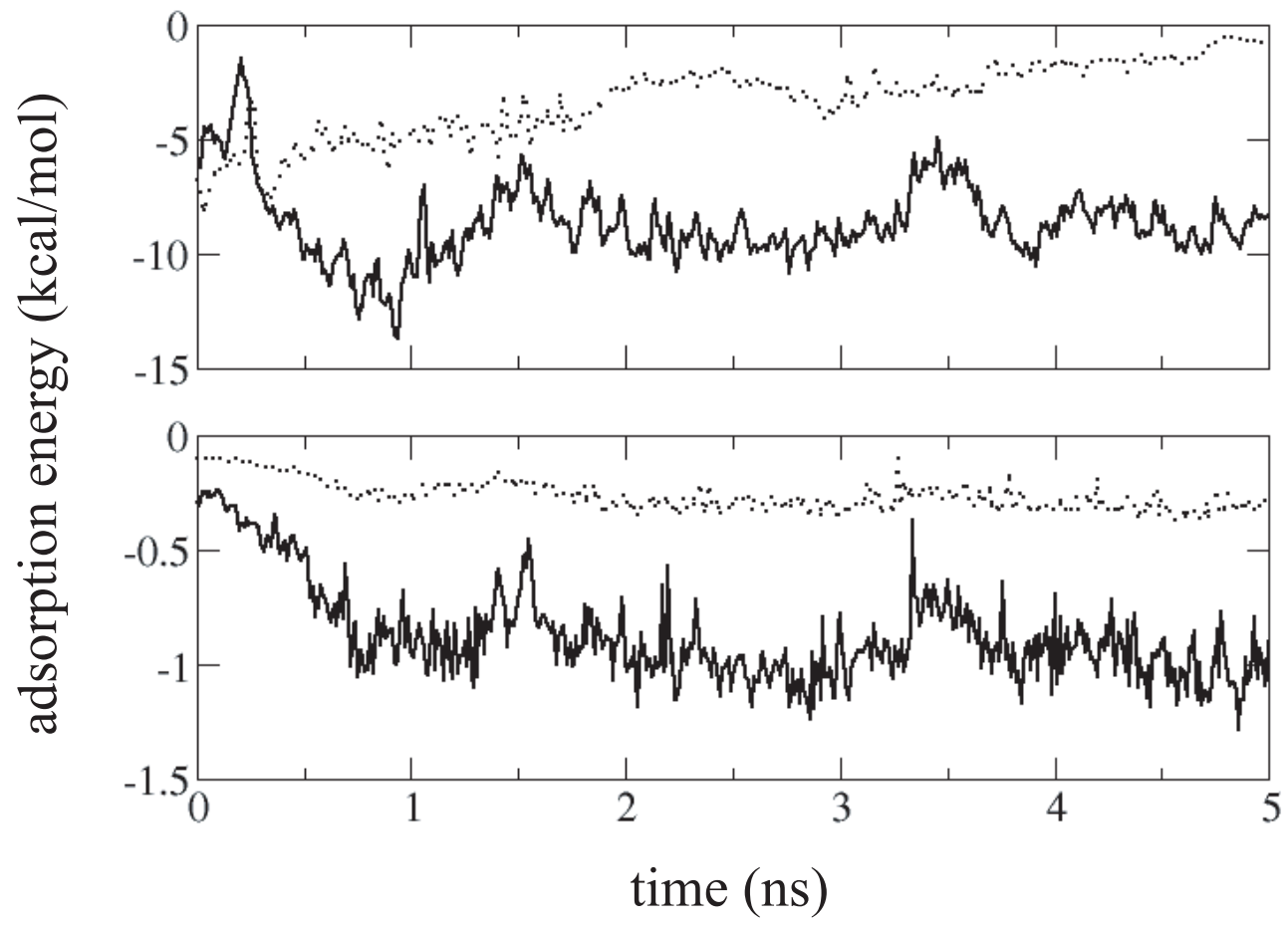

(a)

(b) 

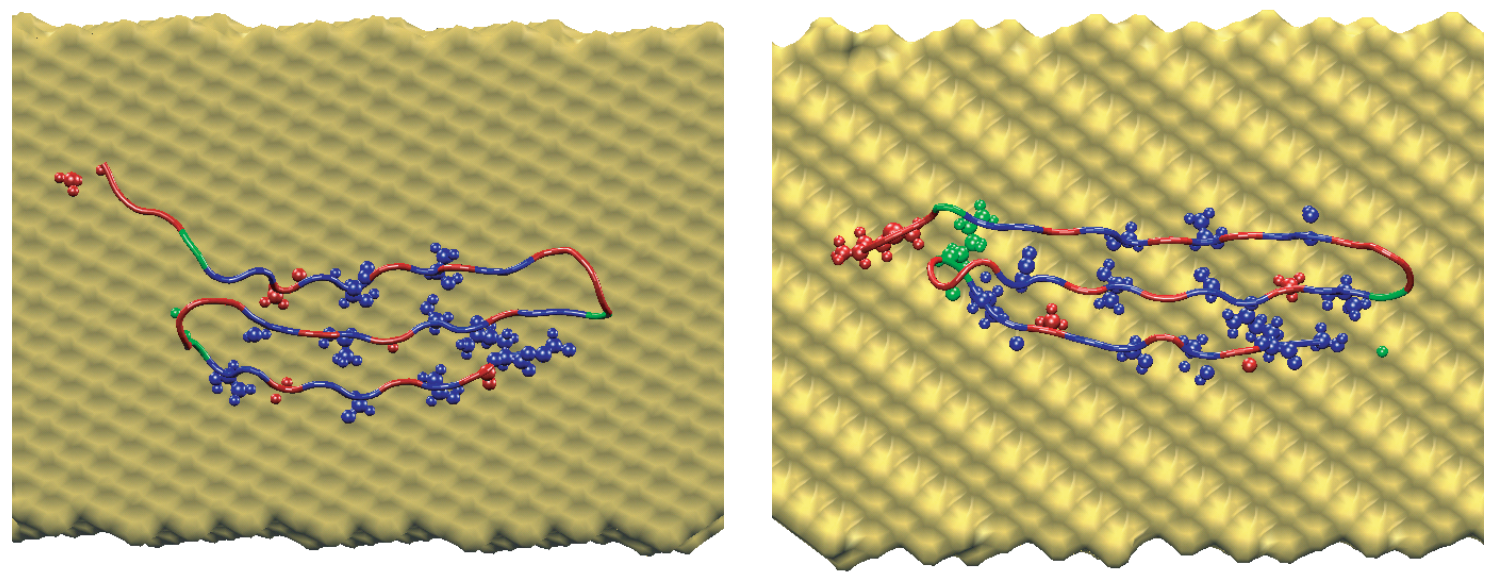

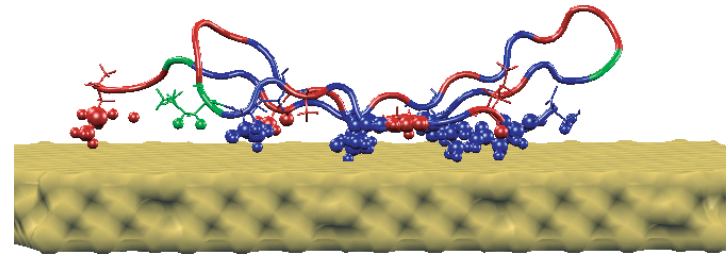

(a)



(b)

Braun, Sarikaya \& Schulten. Figure 3. 


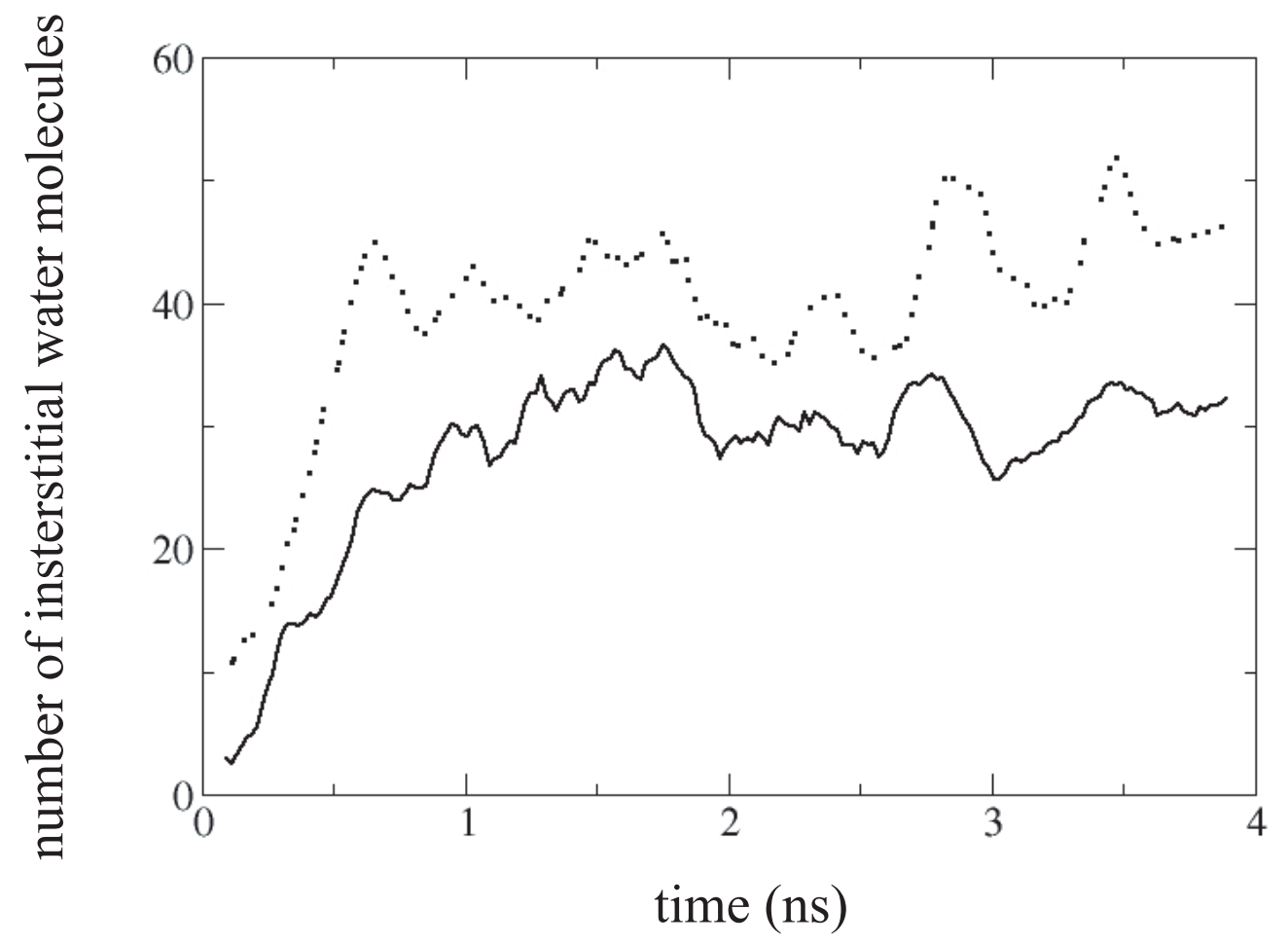

Braun, Sarikaya \& Schulten. Figure 4. 


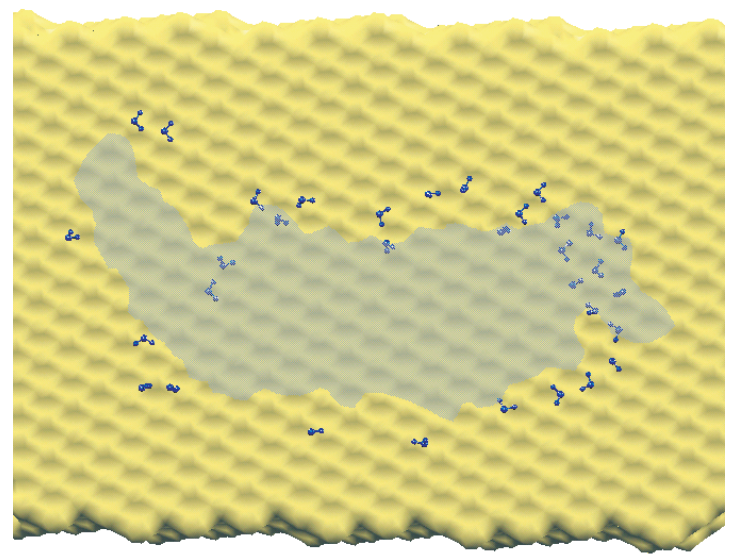

(a)

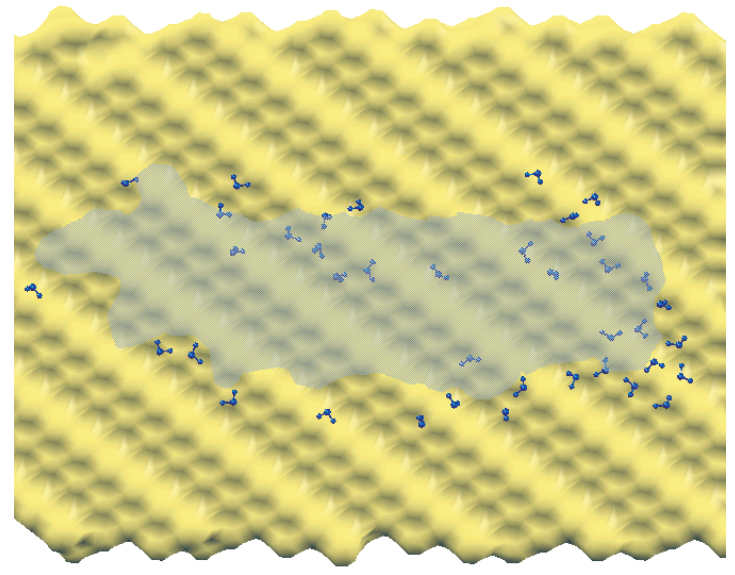

(b)

Braun, Sarikaya \& Schulten. Figure 5. 